\title{
Instrumental requirements for the detection of electron beam-induced object exci- tations at the single atom level in high-resolution transmission electron micro- scopy
}

C. Kisielowski ${ }^{1}$, P.Specht ${ }^{2}$, S. M. Gygax ${ }^{2}$, B. Barton ${ }^{3}$, H. A. Calderon ${ }^{4}$, J. H. Kang ${ }^{5}$, R. Cieslinski ${ }^{5}$

${ }^{1}$ National Center for Electron Microscopy and Joint Center for Artificial Photosynthesis, Lawrence Berkeley National Laboratory, One Cyclotron Rd., Berkeley, CA 94720, USA

${ }^{2}$ Department of Material Science \& Engineering, University of California-Berkeley, Berkeley, CA 94720, USA

${ }^{3}$ Material Sciences Division, Lawrence Berkeley National Laboratory, One Cyclotron Rd., Berkeley, CA 94720, USA

${ }^{4}$ Dept. Fisica, ESFM-IPN, Mexico D.F. 07300, Mexico

${ }^{5}$ The DOW Chemical Company, Midland, MI 48667, USA

\begin{abstract}
This contribution touches on essential requirements for instrument stability and resolution that allows operating advanced electron microscopes at the edge to technological capabilities. They enable the detection of single atoms and their dynamic behavior on a length scale of pico-meters in real time. It is understood that the observed atom dynamic is intimately linked to the relaxation and thermalization of electron beam-induced sample excitation. Resulting contrast fluctuations are beam current dependent and largely contribute to a contrast mismatch between experiments and theory if not considered. If explored, they open the possibility to study functional behavior of nanocrystals and single molecules at the atomic level in real time.
\end{abstract}




\section{Introduction}

With the successful conclusion of DOE's TEAM Project a new generation electron microscopes is available that pushes aberration correction to physical limits [1]. Moreover, it is now apparent that resolution and sensitivity are tradeoffs between the complex technology to correct for chromatic \& spherical lens aberrations and instrument stability $[2,3]$. In the TEAM 0.5 microscope this balance is explored by minimizing the impact of hardware components that are needed to improve on aberration correction. For example, a Cs corrector makes use of only 30-40 additional power supplies while Cc/Cs correction increases this number beyond 150 and adds $\sim 0.5 \mathrm{~m}$ to the column length. In addition TEAM 0.5 makes use of a monochromator in combination with an Ultra-Twin lens [4-7] with its low intrinsic aberrations to obtain outstanding resolution while gaining stability. The instrument is tuned to operate at variable dose rates and voltages [5] and enables observing the relaxation of electron beam-induced sample excitations in real time with single atom sensitivity $[8,9]$.

From a scientific point of view, relaxation and thermalization processes become central scientific aspects if one aims at tracking functionality with single atom sensitivity at atomic resolution [9]. The occurrence of chemical reactions at turn-over frequencies around one second may serve as an example. Previously, contrast variations that are linked to the thermalization of sample excitation were commonly ignored with the exception of pioneering ultrafast experiments using a U-TEM (ultrafast transmission electron microscopy) or a D-TEM (dynamic transmission electron microscopy) [10, 11]. Complementing such dynamic experiments by moving towards atomic resolution, references 8 and 9 recently show that dose rate variations at atomic resolution allow modulating relaxation and thermalization processes while maintaining single atom sensitivity. It is now understood that the relaxation of electron beam-induced excitations can reduce the image contrast by a factor of 2 or more in HRTEM (high-resolution transmission electron microscopy) images [9], which explains the existing discrepancy between experiment and theory that is widely known as the "Stobbs factor" [12]. This contrast loss is forced by the desire to achieve deep sub-Ångstrom resolution with single atom sensitivity in single images, which requires the irradiation of individual atoms with beam currents of 
pico or even nano Amperes per square Ångstrom [7]. From this point of view one can argue that electron beam-induced atom dynamics contribute at least as much to the contrast in a high-resolution image as static scattering contributions do [9]. On the other hand, this situation also provides the opportunity to control and explore reversible or irreversible sample excitations using the electron beam itself as a stimulus in experiments that target specific sample excitations or aim at maintaining pristine object structures of radiation sensitive matter if dose-rates are drastically reduced. In low dose rate conditions the recorded images are dominated by noise. However, a restoration of the electron exit wave function from focal series of tens or hundreds of images can be used to compensate for the information loss in single images [9]. Indeed, preliminary experiments suggest that a rich variety of novel applications can be pursued if relaxation effects are considered including a quantitative measurement of single particle transformation trajectories [11] or diffusion measurements with single atom sensitivity [12, 13]. Most appealing are emerging prospects for imaging pristine catalyst surfaces or the atomic structure of single molecules [14,15]. However, traditional practices for the acquisition of phase contrast images must be altered and advanced electron microscopy equipment must be available. This paper comprehensively describes critical requirements for advanced electron microscopes that must be met to enable probing the dynamic behavior of matter at atomic resolution with single atom sensitivity.

\section{Microscope stability and resolution}

The Figure 1 depicts a typical measurement of apparent focus values from a focus series of images recorded with the TEAM 0.5 microscope. Such measurements are integral part of refining the reconstructed electron exit wave function in complex HRTEM [16-18]. It is essential to recognize that they reflect a convolution of microscope and sample stability since any deviation from linearity is caused by fluctuating lens aberrations or any other electrical or mechanical instability during the measurement time. It is seen in Figure 1 that an extraordinary linearity is achieved even if apparent focus values change by less than $1 \mathrm{~nm}$ per image. As a result of such stability, abrupt focus offsets as small as 3 - $5 \AA$ become visible in Figure 1, which typically occur if insulating sam- 
ples are imaged that moderately charge and de-charge during observation. It is instructive to compare apparent focus values of image series recorded with $0.045 \mathrm{~nm}$ resolution in the TEAM 0.5 instrument [5, Fig. 6] with those recorded in the One Angstrom Microscope (OAM) [19, Fig. 3a] that achieved 0.09 nm resolution in 2000 [20, 21]. One finds that deviations from targeted focus changes are about $0.2 \mathrm{~nm}$ in TEAM 0.5 while they can exceed $10 \mathrm{~nm}$ in the OAM. Thus, instrument stability and measurement precision increased about 50 times between both generations of instruments.

As a result, sample displacements of only a few Ångstroms along the beam direction become relevant and detectable with the TEAM 0.5 instrument [22]. Within the traditional framework of partial coherence and linear imaging, sample displacements should not exceed the lateral resolution of $0.045 \mathrm{~nm}$ in the TEAM 0.5 to exploit the instrument resolution. Moreover, sample displacements in beam direction should be compared with the focal spread $\Delta$ of the electron beam, which is commonly assumed to set the information limit resolution $\mathrm{d}_{\Delta}$ in phase contrast microscopy. In aberration corrected microscopes the point-to-point resolution approaches the information limit resolution, which is given by [23]:

$\mathrm{d}_{\Delta} \approx \sqrt{(\pi \Delta \lambda / 2)}$ with $\Delta=\mathrm{C}_{\mathrm{C}} \sigma(\mathrm{E}) / \mathrm{E}$

Here, $\lambda$ is the wavelength of the electrons, $C_{C}$ is the chromatic lens aberration and $\sigma(E) / E$ is the rms energy spread in the electron beam as a fraction of the total beam energy. Other instabilities are not reflected in equations (1) but reported elsewhere in great detail [23, 24]. Figure 2 shows calculated Contrast Transfer Functions for the TEAM 0.5 microscope at $300 \mathrm{kV}$ and $80 \mathrm{kV}$ of acceleration voltages. It is seen that deep sub-Ångstrom resolution can be maintained throughout the voltage range from $300 \mathrm{kV}$ to $80 \mathrm{kV}$ because of a focal spread of only $0.6 \mathrm{~nm}$ that is largely due to the utilization of a monochromator in combination with a lens of low intrinsic aberrations.

Beyond the traditional treatment of information limit resolution, Haider et al. recently pointed out that an image spread $v$ significantly contributes to limiting the information transfer, too [24]. Image spread $v$ and focal spread $\Delta$ together set the information limit $d$ to be: 
$\left.d^{2} \approx 2\left(v^{2} / \lambda^{2} \Delta^{2}\right) \sqrt{\left(1-0.5 \ln \left(1 / e^{2}\right)\left(\lambda \Delta / \pi v^{2}\right)^{2}\right.}-1\right)$

Typically, the image spread $v$ exceeds tenth of picometers in high performance microscopes and can be measured by tilting the illumination [24]. Figure 3 shows Zemlin tableaus from a $3 \mathrm{~nm}$ thin carbon film that are recorded in this manner. A tilt angle of 78 mrad is uncommonly large and probes for information transfer to $0.5 \AA$ at $80 \mathrm{kV}$. Figure 3 a) describes the TEAM 0.5 performance with the monochromator switched off. In this case a "cigar" shaped damping of the contrast occurs at all depicted tilt angles, which reveals a resolution limiting image and focus spread caused by a $0.9 \mathrm{eV}$ energy width of the Schottky field emitter. In contrast, the effect is absent from Figure $3 \mathrm{~b}$ ) since a monochromatic beam with energy width of less than $100 \mathrm{meV}$ is used for imaging [6,7]. Further, the experiment uncovers the presence of residual lens aberrations at large scattering angles that are typical for the first generation hexapole $\mathrm{C}_{\mathrm{S}}$-correctors. Uhlemann et al. recently pointed out that an image spread of $15-25 \mathrm{pm}$ imposes a hard limit on the resolution in $\mathrm{C}_{\mathrm{C}^{-}}$and $\mathrm{C}_{\mathrm{S}^{-}}$-corrected systems [2] like the TEAM I microscope [1]. They suggest an excitation of thermal magnetic field noise as the cause for a finite irremovable image spread that scales with the corrector length. Consequently, a shorter corrector allows for higher resolution and better stability. The absence of chromatic damping effects in Figure $3 \mathrm{~b}$ ) and the superior $0.45 \AA$ resolution of TEAM 0.5 support their view.

In addition to resolution improvements, the monochromator enables controlling the number of accelerated electrons at the source of the microscope itself and to vary dose rates conveniently while maintaining the extraordinary coherence of an electron beam that is monochromatic to an energy width of $100 \mathrm{meV}$ or less. For this purpose, a Nelsonian illumination scheme is developed $[6,7]$ that bypasses the traditional setup of the condenser system. The beam is dispersed and focused directly onto the sample forming the elongated caustic that is shown in Figure 4a). The width of the illumination is set to match the width of the CCD camera. A monochromator slit is then used to restrict the perpendicular part of the monochromator caustic to a similar dimension so that the size of the illuminated area matches the size of the CCD camera. As a result, electrons from 
the source are only delivered to the imaged area and nowhere else, which is a necessity if one aims at controlling electron beam-induced sample excitations [7]. Moreover, the monochromator caustic can be rapidly shifted electronically, which allows changing dose rates by simply centering different parts of the dispersed electron beam onto the camera. In this manner, dose rates can be altered by many orders of magnitude (Fig. 4a) while the voltage of the incident electron beam is marginally altered by less then 10 $\mathrm{V}$ (Figure $4 \mathrm{~b}$ ). In addition, the change of lens aberrations is predictable and commonly smaller than the precision as to which absolute aberration values can be measured (Fig. $4 b)$.

\section{Wave propagation and numerical aberration correction}

High resolution images that are recorded in microscopes of extraordinary performance reveal an unusual amount of detail since image pattern changes are already observable if the focus changes by only a few Ångstrom. As a result minor shape variations of the imaged objects cause significant pattern changes in images as shown in the defocused image of the gold bridge in Figure $5 \mathrm{a}$ ), which was recorded at $300 \mathrm{kV}$ of acceleration voltage with TEAM0.5. The bridge is less than $4 \mathrm{~nm}$ thick. Any voltage reduction amplifies the effect dramatically such that homogeneous patterns can hardly be recorded from samples with typical shapes at $20 \mathrm{kV}$ [5] unless it is a monolayer of light elements such as graphene. Therefore, the interpretation of a recorded image contrast remains challenging even in images from aberration-corrected microscopes unless the samples are prepared atomically flat, remain not tilted during observation and the acceleration voltage is high. These restrictions can be relaxed by a reconstruction of the electron exit wave function from focal series of images $[16,17]$, which generates an aberrationcorrected in-line hologram. For his purpose we use a Gerchberg-Saxton algorithm that is implemented in the McTempas software package [18]. The phase of the reconstructed wave function from an image series of the gold bridge is shown in Figure $5 \mathrm{~b}$ ). Unlike single images the electron exit wave function can be locally refocused from one image plane into another one by multiplying a wave propagator: 
$P=\exp (i x)=\exp \left(2 \pi i\left(1 / 2^{\star} f^{\star} N g^{2}+1 / 4^{*} C_{S}{ }^{*} \lambda^{3} / g^{4}+1 / 6^{*} C_{5}{ }^{*} \lambda^{5} / g^{6}\right)\right)$

in Fourier space to the wave function. Here, $g$ is a spatial frequency and $f=C_{1}, C_{S}=C_{3}$, $\mathrm{C}_{5}$ are the spherical aberration coefficients to fifth order. For $\mathrm{C}_{3}=\mathrm{C}_{5}=0$ a wave propagation by a focal distance $f$ equals the geometrical displacement $z$ of the image away from its initial zero-focus plane in beam direction. If geometrical sample changes are present that are often caused by an unavoidable surface roughness or by the shape of nanocrystals they can now be corrected by local wave propagation to a precision that reaches the Angstrom level. For example, the Figure $5 \mathrm{c}$ ) shows the refocused phase of the electron exit wave function from Figure $5 \mathrm{~b}$ ). Since the center of the depicted gold bridge was refocused by $f=-3.9 \mathrm{~nm}$, the sample thickness changes by an equal value compared to the edge of the sample, which is defocused now. Obviously, procedures that manipulate properties of the contrast transfer function can be pursued to allow for a targeted extraction of sample properties. The approach forms a foundation for electron tomography from single projections [25], which expands current abilities [26] to include nanocrystals.

Equation (3) gives the aberration function $\mathrm{X}$ for finite values of the spherical aberration coefficients, only. It can be generalized to include other aberrations such as coma or two-fold astigmatism to create numerical phase plates [e.g. 27]. Thereby, resolution can be extended by software beyond limits that are set by hardware and are always present, as shown in Figure $3 \mathrm{~b}$ ) for TEAM 0.5 operated at $80 \mathrm{kV}$. Different optimization strategies to correct for residual lens aberrations are proposed [27]. In Figure 6 we show how they can be removed by applying a numerical phase plate in combination with a propagation of amplitudes and phases of reconstructed wave functions. Figure 6 a) shows three experimental images from the focal series of the gold bridge. An elongation of the atom columns is most prominent in the image recorded at a nominal (calculated) focus of $17 \mathrm{~nm}$ that is caused by a residual 2 -fold astigmatism of $3.4 \mathrm{~nm}$. This image is chosen to define the zero-focus plane because it exhibits a minimal amplitude contrast variation, which is characteristic for a focused weak phase object. The aberration is measured and removed during the reconstruction process using numerical phase plates. Successively, images are recalculated from the reconstructed and corrected 
wave function at the same focus values of $34 \mathrm{~nm}, 17 \mathrm{~nm}$, and $-10 \mathrm{~nm}$ to show in Figure $6 \mathrm{~b})$ that the distortion is removed. In fact, only marginal differences appear between the aberration-corrected exit wave function and calculated, aberration-free images using multi-slice simulations as can be seen by comparing Figures $6 b$ and $6 c$. Nevertheless, a wave propagation of the phases still reveals intensity modulations on top of an expected sinusoidal oscillation [28] that are due to minimal lens aberrations (Figure 6d). Such intensity modulations on a length scale of single pixels $(0.015 \mathrm{~nm} / \mathrm{pixel})$ occur in propagated wave functions even if the residual lens aberrations are smaller than needed to achieve the $0.045 \mathrm{~nm}$ resolution of the TEAM 0.5 microscope. Consequently, wave propagation is an extraordinary suitable tool for local aberration corrections on the atom columns themselves. The approach builds on the assumption that single atomic columns must appear circular. Clearly, the outlined post-acquisition aberrationcorrection can be executed beyond the $0.045 \mathrm{~nm}$ information limit of the TEAM $0.5 \mathrm{mi}-$ croscope to extend resolution. The process is most affective at low voltages where scattering angles increase beyond the correctors ability to properly adjust lens aberrations as shown in Figure 3b. However, it is also noted that it becomes challenging to distinguish residual lens aberrations from physically relevant atom displacements on this deep sub-Ångstrom length scale. Numerically, measurements of atom column displacements with 1 pm precision were reported as early as 1998 [29], which already equal half the wavelength of electrons accelerated to $300 \mathrm{kV}$.

\section{Beam-sample interactions and atom motion}

Since early applications of high-resolution electron microscopy it is known that the high energetic electron beam, which is needed to create images with atomic resolution, can strongly modify the observed objects in particular if high brightness electron sources are employed [30]. In fact, maintaining a pristine object structure during an image acquisition is known to be essential and considered an "art". If abandoned it can lead to seemingly endless debates about the "true" atomic structure of matter [31] as it is the case for reports on the indium cluster formation in InGaN [31-34]. The underlying beamsample interactions are most obvious if small nanocrystals are observed [35]. It was 
reported that electron beam-induced sample damage is easily recognizable at surface sites where binding energies are lowered and the displacement, ionization or evaporation of atoms occurs most frequently [36,8]. Unfortunately, it is a persistent misconception that bulk binding energies are relevant in such processes if acceleration voltages between $80 \mathrm{kV}$ and $300 \mathrm{kV}$ are used [e.g. 33].

Beam-sample interactions are sample size and beam current dependent, which can be understood in the following manner: Suppose a scientific task includes measuring a sample size distribution. For this purpose, low magnification bright-field images could be recorded as shown in Figure 7a). Imaging at low magnification implies that the beam current per irradiated area is low. In this case object alterations at the atomic scale are not observable because they may either happen rarely or cannot be detected. Therefore, the objects appear stable in the electron beam. Such measurements typically result in producing size distributions as depicted in Figure $7 \mathrm{~b}$ ). Less recognized is the fact that such distributions also depict a large variation of the total binding energies of the imaged particles. A simple but reasonable estimate is also in Figure $7 \mathrm{~b}$, which assumes a typical binding energy of $\sim 4 \mathrm{eV} /$ atom, an atom diameter of $\sim 0.3 \mathrm{~nm}$ and a material crystallization in a cubic structure. It is seen that an increase of the particle size from 3 $\mathrm{nm}$ to $6 \mathrm{~nm}$ rapidly increases the total binding energies of the three-dimensional objects from 5,000 eV to $25,000 \mathrm{eV}$. Even though such energy values can be considered large, it is helpful to compare them to the electrical power equivalent $\mathrm{P}=\mathrm{U}^{*} \mathrm{I}$ that is delivered by the electron beam within one second of exposure time during observation. An acceleration voltage of $80,000 \mathrm{~V}$, for example, would require an extreme reduction of beam currents to zepto $\left(10^{-21}\right)$ Amperes to match the binding energy of small particles (Figure 7 b)! Even if one assumes that only 0.01 percent of the incident beam current is absorbed in the sample, they are in the range of atto or femto Amperes to guarantee maintaining a pristine crystal structure during the observation. Unfortunately, atomic resolution imaging requires exposing the samples to beam currents that are by orders of magnitude larger [7, 35]. Consequently, the structural integrity of small nanocrystals is by default compromised during their observation at high magnification. As a result, Oswald ripening is commonly observed during such experiments since the small particles disintegrate first and the created atoms tend to diffuse across surfaces and attach to 
larger particles as shown in Figure 7c). Noteworthy, Simonsen et al. [37] reported the observation of Oswald ripening by in situ transmission electron microscopy at $650{ }^{\circ} \mathrm{C}$ using low current densities. They also noted that a narrow particle distribution retards the ripening process as one would expect since the total particle energy equalizes. Beyond doubt this experiment highlights the intimate relation between electron beam excitations and temperature increase.

From this point of view it seems somewhat astounding that atomic resolution imaging is applied with great success over decades. These puzzling circumstances find a natural explanation by recent research suggesting that electron beam-induced sample excitations are indeed common but that they are usually reversible $[8,9,38]$. Thereby, atom loss or structural damage are greatly retarded. Insight into beam-sample interactions at the atomic scale is enabled by the development of high performance microscopes. Only such instruments allow tracking single atom motion on a length scale between a few picometer and a few Angstroms that is caused by the relaxation or thermalization of electron beam-induced sample excitations. A first real time study of electron beam-induced single atom excitations is published in Reference 8 for a rhodium catalyst. Figure 8 a) shows snapshots of movies related to this investigation, which are provided as supplementary information. The depicted image simulation of a wedge shaped sample includes atom displacements between $30 \mathrm{pm}$ and $50 \mathrm{pm}$ in 3 dimensions (frozen phonons in 3D) that are much larger than one would expect from Debye Waller factors. It is argued that such large atom displacements from their equilibrium sites are a result of rare events that reflect collective, reversible atom excitations into meta-stable configurations of higher total energy [9]. From Figure 8 a) and the supplementary movies it is seen that the simulated and measured contrasts of the moving atoms match for a crystal thickness of 2-3 atomic layers.

Similarly, the Figure 8 b) shows a phase image of a multi-wall carbon nanotube with attached citric acid molecules that was reconstructed from a focus series of 40 images using the TEAM I microscope [39]. It is seen that a $0.21 \mathrm{~nm}$ atom column spacing of the carbon nanotube is well resolved. In contrast, the images of the attached molecules are blurred to a width of $\sim 0.5 \mathrm{~nm}$ because of electron beam-induced molecule vibrations that affect each image of the series differently to cause the blur in the reconstruction. 
Since both resolution results are recorded simultaneously it is clear from Figure 8 b) that the blur in the image of the molecules is caused by beam induced molecule motion and nothing else.

For atom displacements smaller than $50 \mathrm{pm}$, however, such an assignment may not be unique because the images can be affected by instrument instabilities below the resolution limit. A distinction between both scenarios is challenging but possible by dose rate variations [9], which change the excitation of vibrations without affecting the instrument performance.

\section{Outlook}

Electron microscopy with variable dose rates is expected to develop rapidly because it becomes a key experiment that allows stimulating and controlling system dynamics at atomic resolution with single atom sensitivity. As an example we show in Figure 9 that the time evolution of an amorphous monolayer can be studied in real time to observe the restructuring of atom arrangements and single bonds. In this case it is seen that a graphene structure emerges from a random distribution of hydrocarbon atoms within 4 seconds of the experiment. It proves that graphene is a more stable structure than an amorphous arrangement of the visible carbon atoms. Ultimately, such experiments can contribute to better understand functional behavior of matter if more complex systems were considered. The low dose rate approach is in particular advantageous in combination with environmental electron microscopy, since it allows maintaining atomic resolution and single atom sensitivity at finite pressure and temperature to probe for catalytic processes [40]. Limits to this technology are currently unexplored but do exist since irreversible atom displacements occur inevitably.

\section{Acknowledgements}

Electron Microscopy was performed at the NCEM, which is supported by the Office of Science, Office of Basic Energy Sciences of the U.S. Department of Energy under Contract No. DE-AC02-05CH11231. Experiments with NiFeOx catalysts were performed for the Joint Center for Artificial Synthesis, a DOE Energy Innovation Hub, supported 
through the Office of Science of the U.S. Department of Energy under Award Number DE-SC0004993. The Dow Chemical Company supported P. Specht for the investigation of the rhodium catalysts.

\section{References}

[1] U. Dahmen, R. Erni, V. Radmilovic, C. Kisielowski, M.-D. Rossell, P. Denes, Phil. Transact. Royal Soc. A 367, 3795 (2009)

[2] S. Uhlemann, H. Müller, P. Hartel, J. Zach, M. Haider, Phys. Rev. Lett. 111, 046101 (2013)

[3] S. M. Schramm, S. J. van der Molen, R. M. Tromp, Phys. Rev. Lett. 109, 163901 (2012)

[4] C. Kisielowski, B. Freitag, M. Bischoff, H. van Lin, S. Lazar, G. Knippels, P.

Tiemeijer, M. van der Stam, S. von Harrach, M. Stekelenburg, M. Haider, S. Uhlemann, H. Mueller, P. Hartel, B. Kabius, D. Miller, I. Petrov, E. A. Olson, T. Donchev, E. A. Kenik, A. R. Lupini, J. Bentley, S. J. Pennycook, I. M. Anderson, A. M. Minor, A. K. Schmid, T. Duden, V. Radmilovic, Q. M.Ramasse, M. Watanabe, R. Erni, E. A. Stach, P. Denes, U. Dahmen, Microscopy \& Microanalysis 14, 469, (2008)

[5] B. Barton, B. Jiang, C. Y. Song, P. Specht, H. A. Calderon, C. Kisielowski, Microscopy \& Microanalysis 18, $982(2012)$

[6] P.C. Tiemeijer, M. Bischoff, B. Freitag, C. Kisielowski, Ultramicroscopy 118, 35 (2012)

[7] P.C. Tiemeijer, M. Bischoff, B. Freitag, C. Kisielowski, Ultramicroscopy 114, 72 (2012)

[8] P. Specht,R. J. Gulotty, Jr.; D. Barton, R. Cieslinski, S. Rozeveld, J. H. Kang, O. D. Dubon, C. Kisielowski, ChemCatChem 3, 1034 (2011)

[9] C. Kisielowski, L.-W. Wang, P. Specht, H. A. Calderon, B. Barton, B. Jiang, J. H. Kang, R. Cieslinski, Phys Rev. B 88024305 (2013)

[10] D. J. Flannigan, A. H. Zewail, Acc. of Chem. Res. 45, 1828 (2012)

[11] B. W. Reed, M. R. Armstrong, N. D. Browning, G. H. Campbell, J. E. Evans, T. B. LaGrange, D. J. Masiel, Microscopy \& Microanalysis 15, 272 (2009)

[12] M.J. Hÿtch, W.M. Stobbs, Ultramicroscopy 53, 191 (1994)

[11] H. Zheng, J. B. Rivest, T. A. Miller,B. Sadtler, A. Lindenberg, M. F. Toney, L.-W. Wang, C. Kisielowski, A. P. Alivisatos, Science 333, 206(2011)

[12] H. Zheng, B. Sadtler, C. Habenicht, B. Freitag, A. P. Alivisatos, C. Kisielowski, Ultramicroscopy 134, 207, 2013

[13] D. Alloyeau, B. Freitag, S. Dag, L.-W. Wang, C. Kisielowski, Phys. Rev. B 80, 014114 (2009)

[14] B. Barton, R. Buonsant, A. Dong, D. Milliron, L. Hansen, S. Helveg, B. Jiang and C. Kisielowski, Microscopy \& Microanalysis 18, 1606 (2012)

[15] P. Specht, D. Barton, J. Kang, R.C. Cieslinski, O.D. Dubon, C. Kisielowski, Microscopy \& Microanalysis 17, 1064 (2011)

[16] W. Coene, A. Thust, D. Van Dyck, M. Op de Beeck, Ultramicroscopy, 64, 109 (1996)

[17] W. K. Hsieh, F. R. Chen, J.J.Kai, A. I.Kirkland, Ultramicroscopy 98, 99 (2004) 
[18] http://www.totalresolution.com/

[19] T. Bartel, J. R. Jinschek, B. Freitag, P. Specht, and C. Kisielowski, Quantitative electron microscopy of InN-GaN alloys, phys. stat. sol. (a) 203 (2006) $167-175$ [20] C. Kisielowski, C. J. D. Hetherington, Y. C. Wang, R. Kilaas, M. A. O'Keefe, A. Thust, Ultramicroscopy 89, 243 (2001)

[21] C. Kisielowski, A. Schwartzmann, C. E. Nelson, C. Song, R. Kilaas, A. Thust, Microscopy \& Microanalysis 6, 14, (2000)

[22] C. Kisielowski, P. Specht, D. Alloyeau, R. Erni, Q. M. Ramasse, Frontiers of Characterization and Metrology for Nanoelectronics, D.G. Seiler, A.C. Diebold, R. McDonald, C.M. Garner, D. Herr, R.P. Khosla, E.M. Secula (eds.), American Institute of Physics Conference Proc. 1173, 231 (2009)

[23] M. A. O'Keefe. Ultramicroscopy 47, 282 (1992)

[24] M. Haider, P. Hartel, H. Müller, S. Uhlemann, J. Zach, Microscopy \& Microanalysis 16, 393 (2010)

[25] F.R. Chen, C. Kisielowski, D. Van Dyck, Nature Com. in review (2014)

[26] D. Van Dyck, J.R. Jinschek, F.R. Chen, Nature 486, 243 (2012)

[27] R. Erni, M. D. Rossell, P. N.H. Nakashima, Ultramicroscopy 110, 151 (2009)

[28] A. Wang, F. R. Chen, S. Van Aert, D. Van Dyck, Ultramicroscopy 110, 527 (2010)

[29] Kisielowski, C.; Schmidt, O.; Yang, J.; Atomic Scale Aluminum and Strain Distribution in a GaN/AlxGa1-xN Heterostructure, Mat. Res. Soc. Symp. 482, 369 (1998)

[30] B. Freitag, G Knippels, S. Kujawa, P.C. Tiemeijer, M. Van der Stam, D. Hubert, C. Kisielowski, P. Denes, A. Minor and U. Dahmen, M. Luysberg, K. Tillmann, T. Weirich (Eds.), EMC 1 Instrumentation and Methods 55-56, (2008)

[31] M. Smeeton, M. J. Kappers, J. S. Barnard, M. E. Vickers, and C. J. Humphreys, Appl. Phys. Lett. 83, 5419 (2003)

[32] T. P. Bartel, P. Specht, J. C. Ho, C. Kisielowski, Phil. Mag. 87, 1983 (2007)

[33] K.H. Baloch, A. C. Johnston-Peck, K. Kisslinger, E.A. Stach, S. Gradecak, Appl.

Phys. Lett. 102, 191910 (2013)

[34] C. Kisielowski, Comment to Appl. Phys. Lett. 102, 191910 (2013), submitted 2014

[35] D. J. Smith, A. K. Petford-Long, L. R. Wallenberg, J. O. Bovin, Science 233, 872 (1986)

[36] R. M. Wang, O. Dmitrieva, M. Farle, G. Dumpich, H. Q. Ye, H. Poppa, R. Kilaas, C. Kisielowski, Phys. Rev Lett. 100, 017205 (2008)

[37] S. B. Simonsen, I. Chorkendorff, S. Dahl, M. Skoglundh, J. Sehested, S. Helveg, J. Am. Chem. Soc., 132, 7968 (2010)

[38] K. Kim, S. Coh, C. Kisielowski, M. F. Crommie, S. G. Louie, M. L. Cohen, and A. Zettl, Nature Comm. 4, 2723 (2013)

[39] S. J. Haigh, B. Jiang, D. Alloyeau, C. Kisielowski, A. I. Kirkland, Ultramicroacopy 133, 26 (2013)

[40] J. R. Jinschek and S. Helveg, Micron 43, 1156 (2012). 


\section{Figures}

Figure 1:

Measured focus values from a series of 50 images of $\mathrm{MgO}$ nano-crystals in [100] orientation as shown by the Fourier transform of the wave function in the inset.

The image series was recorded at $300 \mathrm{kV}$ in the TEAM 0.5 microscope. A linear regression to the data reveals abrupt focus offsets marked by arrows that are caused by moderate charging of the semi-insulating sample. Such offsets in electron beam direction are only 3-5 $\AA$ large but clearly detectable because of the extraordinary stability of the microscope.

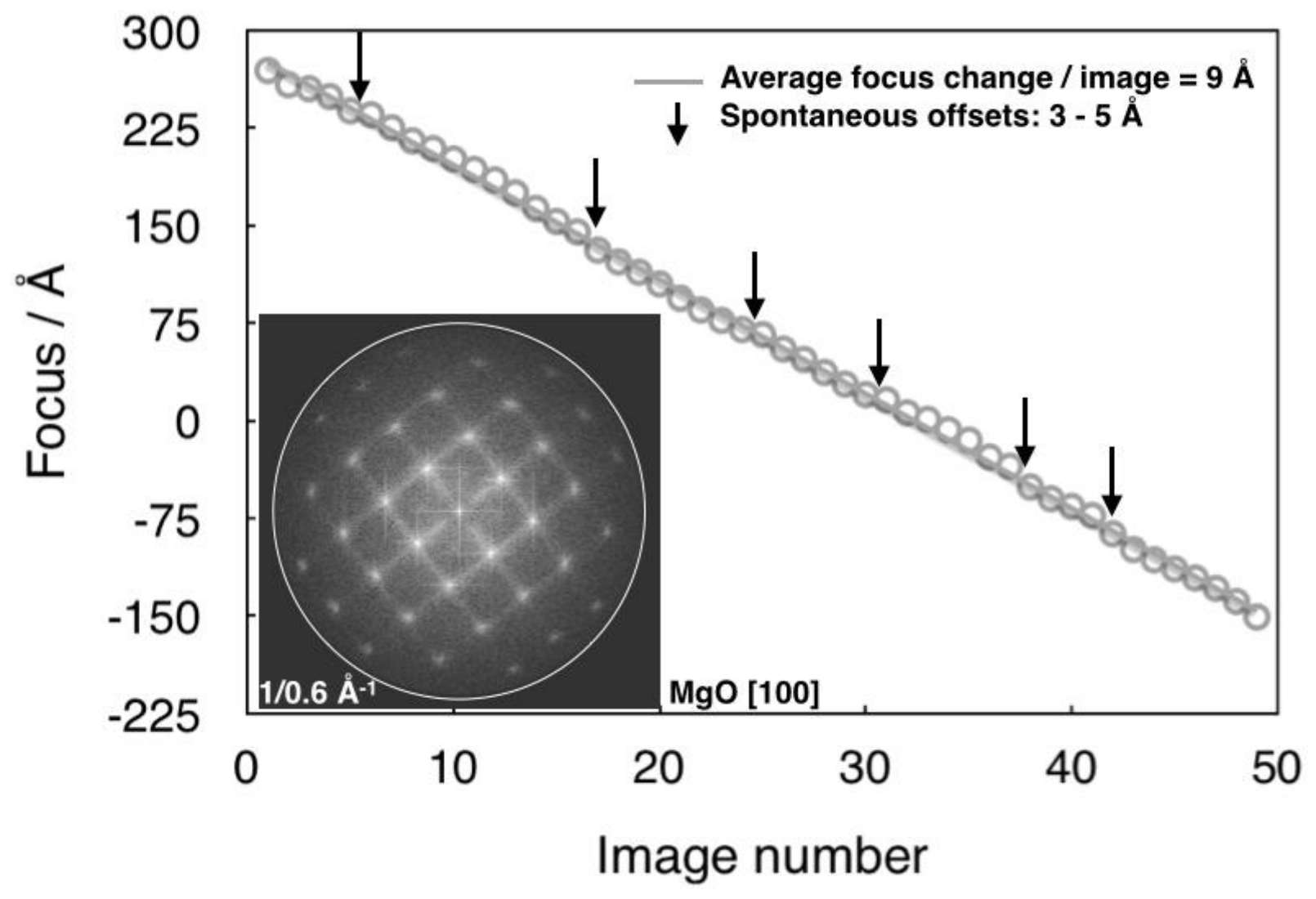


Figure 2:

CTF calculations for the TEAM 0.5 microscope. Intensities drop to 1/e of their maximum at the information limit, which is marked by grey arrows.

a) Information transfer to below $0.05 \mathrm{~nm}$ was reported for $300 \mathrm{kV}[4,21]$, which requires a defocus spread $\mathrm{d} \Delta$ of $0.6 \mathrm{~nm}$ for an energy spread $\Delta \mathrm{E}$ of $\sim 100 \mathrm{meV}$. b) Contrast transfer upon voltage reduction to $80 \mathrm{kV}$ with the monochromator switched on. c) The effect of a monochromatic illumination: Switched off it disables the sub-Ångstrom performance of TEAM 0.5 . The fact that the chromatic aberration $\mathrm{C}_{C}$ of an Ultra-Twin lens is voltage dependent and drops from $\sim 1.4 \mathrm{~mm}$ at $300 \mathrm{kV}$ to $0.7 \mathrm{~mm}$ at $80 \mathrm{kV}$ [5] is not included in the description.

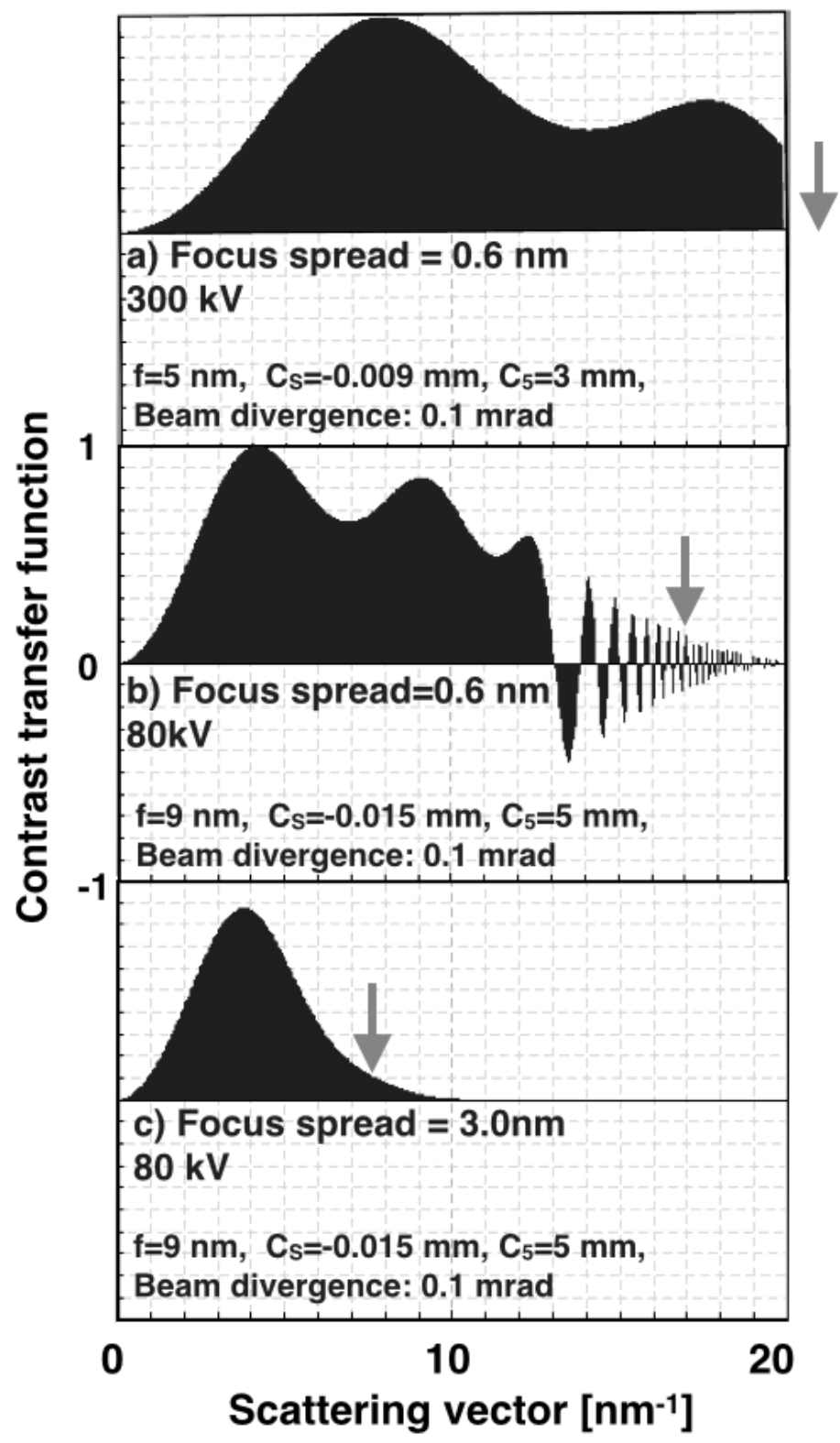


Figure 3:

Focal spread and image spread limit resolution

Tilt series to a largest angle of $78 \mathrm{mrad}$ at $80 \mathrm{kV}$ using the TEAM 0.5 microscope. a) Monochromator switched off. b) Monochromated illumination. The distortions of the Thon rings at large tilt angles measure the residual lens aberrations that cannot be corrected by hardware in the TEAM 0.5 because it is equipped with a hexapod CS-corrector, only.

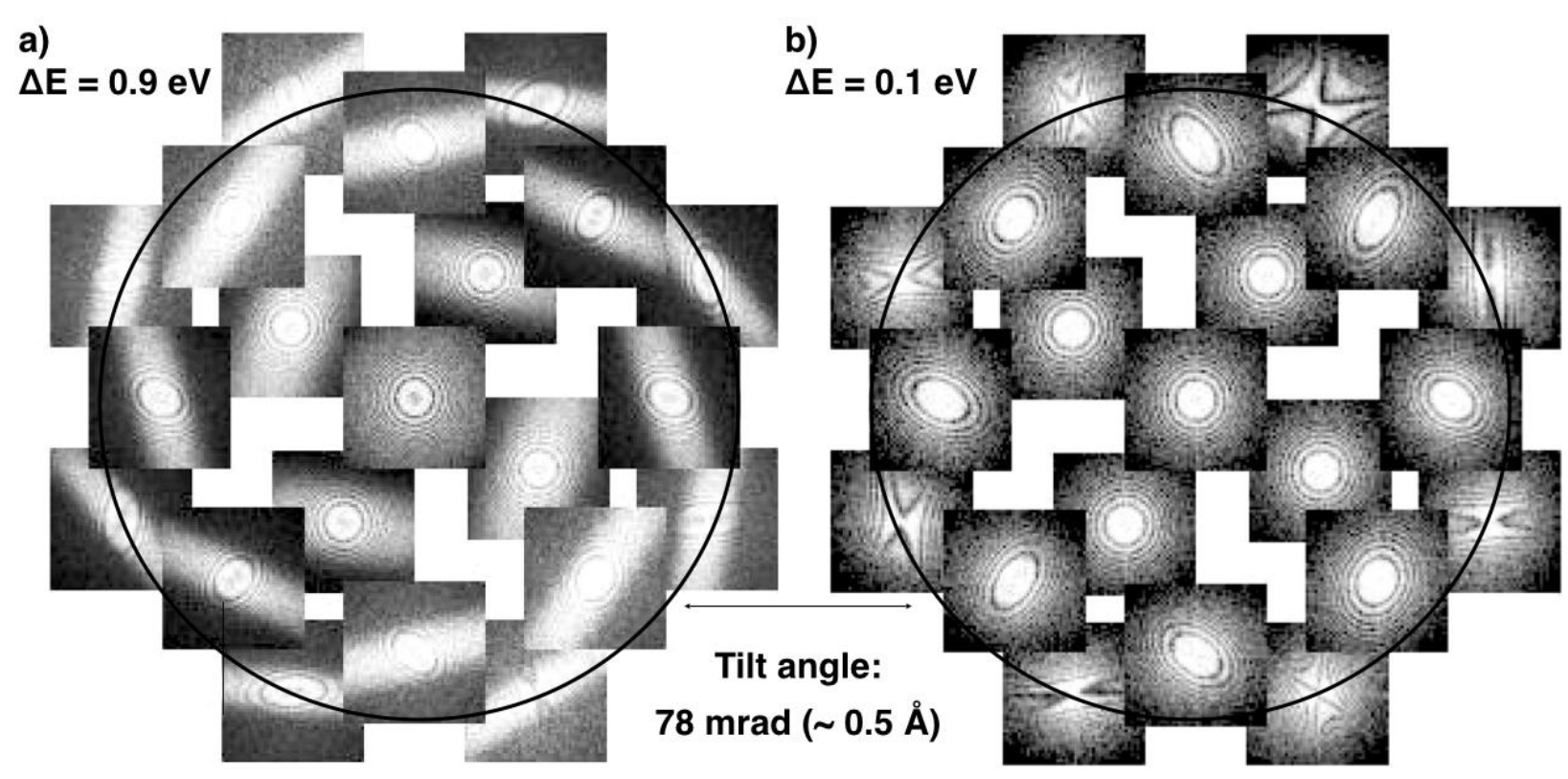


Figure 4:

A Nelsonian illumination scheme

a) Focused and dispersed monochromator caustic at $80 \mathrm{kV}$. A slit in the monochromator and the beam focus are set such that only the CCD camera is illuminated at atomic resolution. Shifting different parts of the monochromator illumination onto the camera allows changing dose rates by orders of magnitude. b) Lens aberrations measured from Zemlin tableaus change with the monochromator shift in a predictable manner $(\mathrm{C} 3, \mathrm{C} 5=$ spherical aberration coefficients of third and fifth order, $\mathrm{B} 2=$ coma, $\mathrm{A} 2=$ threefold aberration). Trends are detectable. Spherical aberration $(\mathrm{C} 3, \mathrm{C} 5)$ coefficients are constant within the errors of the experiments.
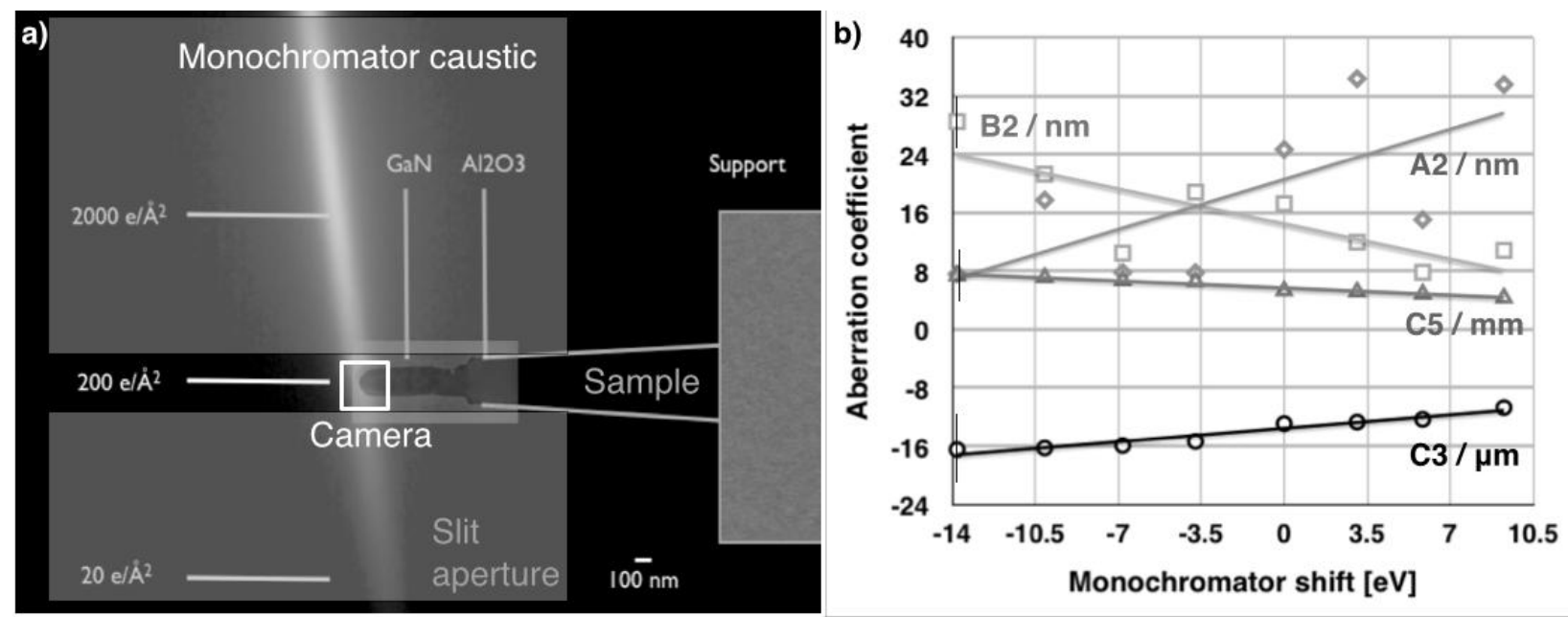
Figure 5:

Wave propagation

a) Defocused image of a gold bridge, TEAM 0.5, $300 \mathrm{kV}$. The sample is dome shaped as a result of specific growth conditions. b) Reconstructed phase of the electron exit wave function from a focus series of 20 images. The wave is focused to the edge of the sample. Unfocused parts of the image exhibit a broad phase distribution that can be donut shaped. c) Phase of the propagated wave function after propagation by - $3.9 \mathrm{~nm}$ to focus the center of the gold bridge.

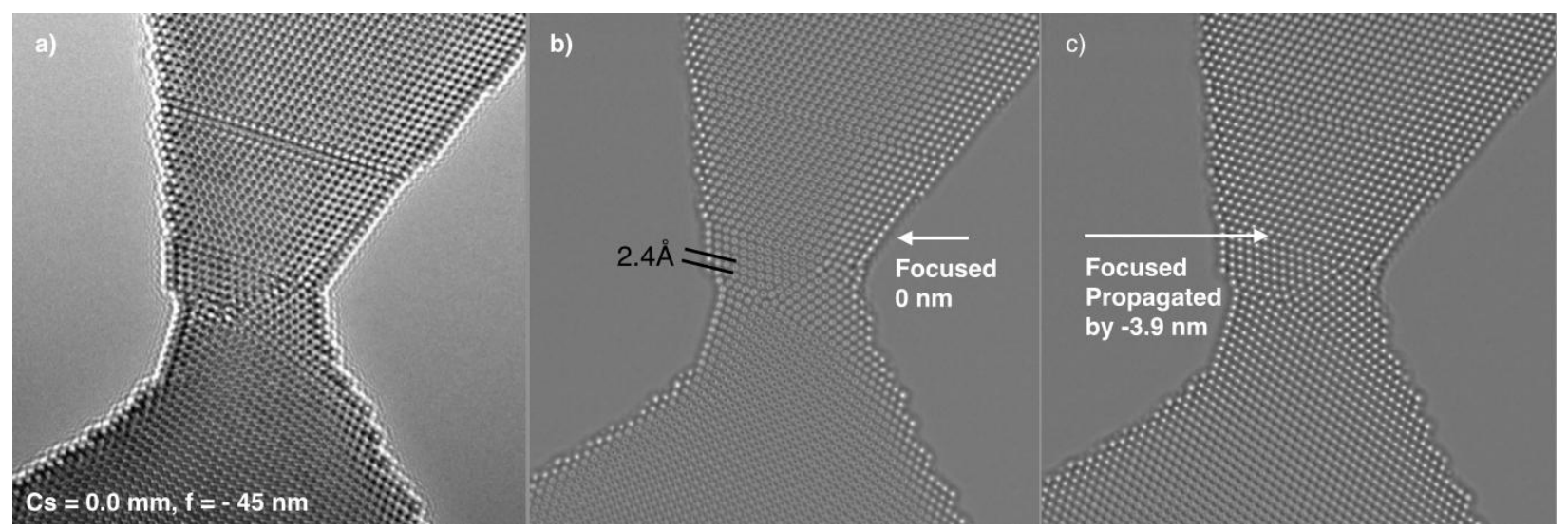


Figure 6:

Approaches to resolution extension

a) Amplitude image of the as-reconstructed edge of the gold bridge from Figure 5 shown for 3 images of the focus series. Atom columns appear elongated because of a two-fold astigmatism as indicated. The distortion can be recognized best at a focus of $17 \mathrm{~nm}$ that is used to define the zero-focus plane (minimum amplitude contrast) b) Images after correction of the two-fold astigmatism. c) Comparison with multi-slice simulations. d) Absolute phase values of a single pixel in the center of a column (size $0.015 \mathrm{~nm}$ ) as a function of propagation distance. Experimental amplitude and phase images of the column are inserted for two propagation distances.
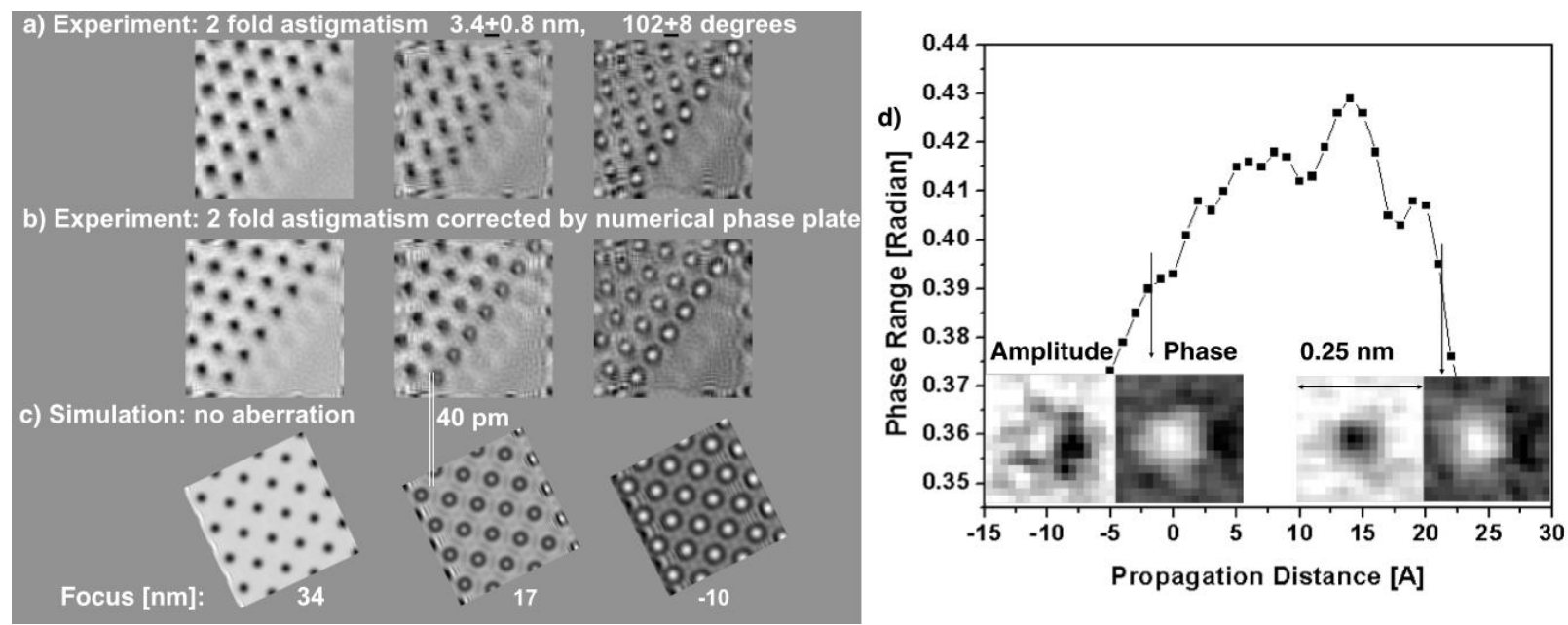


\section{Figure 7:}

\section{Beam sample interactions}

a) Bright field TEM image of $\mathrm{FeNiO}_{x}$ nano particles. b) A calculated particle size distribution together with an estimate of their size dependent binding energy. Current values are given that would be required to match the binding energies of $3 \mathrm{~nm}$ and $6 \mathrm{~nm}$ small particles to the electrical energy that is delivered by the electron beam in one second at $80,000 \mathrm{~V}$. c) High Angle Annular Dark Field image of $\mathrm{FeNiO}_{x}$ nano particles before and after being continuously exposed to a scanning electron beam with $0.1 \mathrm{~nm}$ A beam current for seven minutes. Oswald ripening is observed in the exposed box- shaped area. Particle coarsening is absent from areas surrounding the boxes because they are only exposed twice to the electron beam for 10 seconds to record both images.
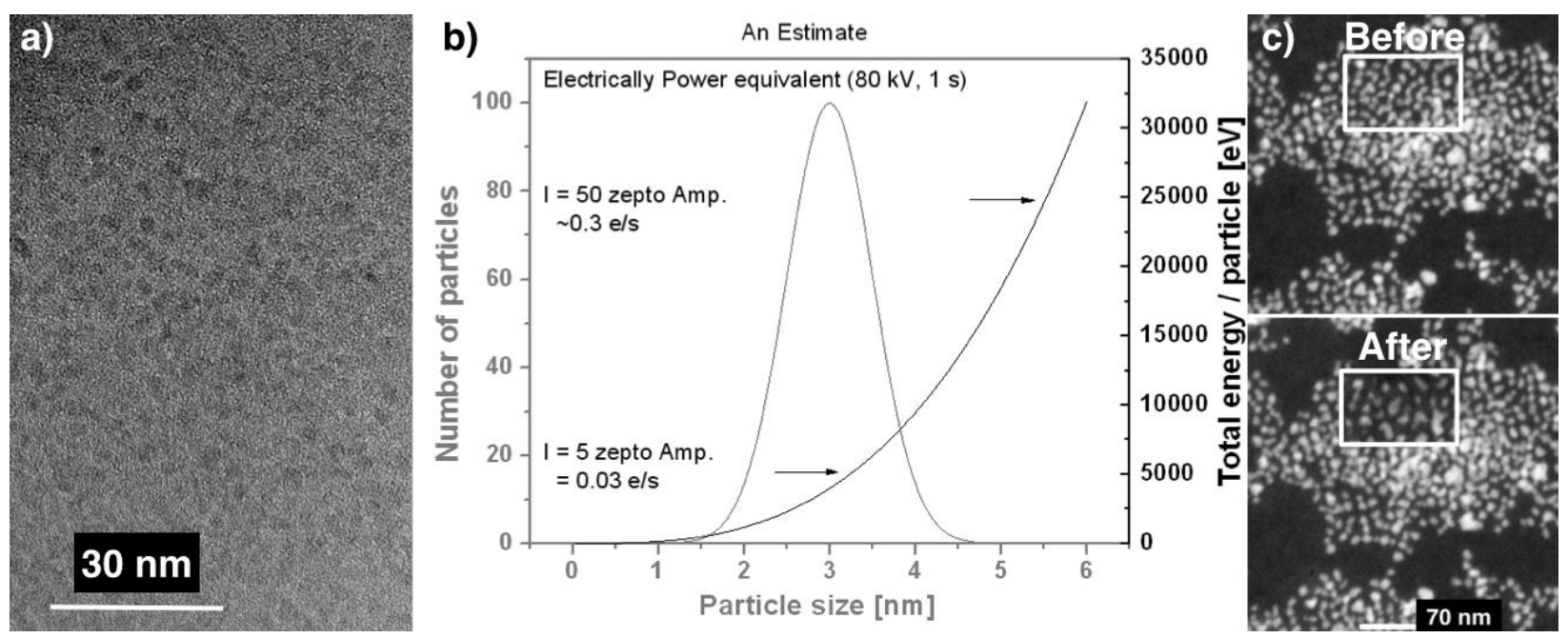


\section{Figure 8:}

Beam induced atom column vibrations

a) Rh atoms in a wedge shaped structure with 1 to 4 atoms per column were calculated in a 3D frozen phonon simulation (top, supplemental movie "Rh motion sim.avi") and compared with the changing contrast of an experimentally observed Rh particle (bottom, supplemental movie "Rh motion 80 kV.avi"). b) Phase image of a multi-wall carbon nanotube (CNT) with attached citric acid molecules. At the walls of the CNT atom column spacing of $0.21 \mathrm{~nm}$ is resolved. The attached molecules, however, are blurred to a width of approx. $0.5 \mathrm{~nm}$ for the same electron beam conditions.
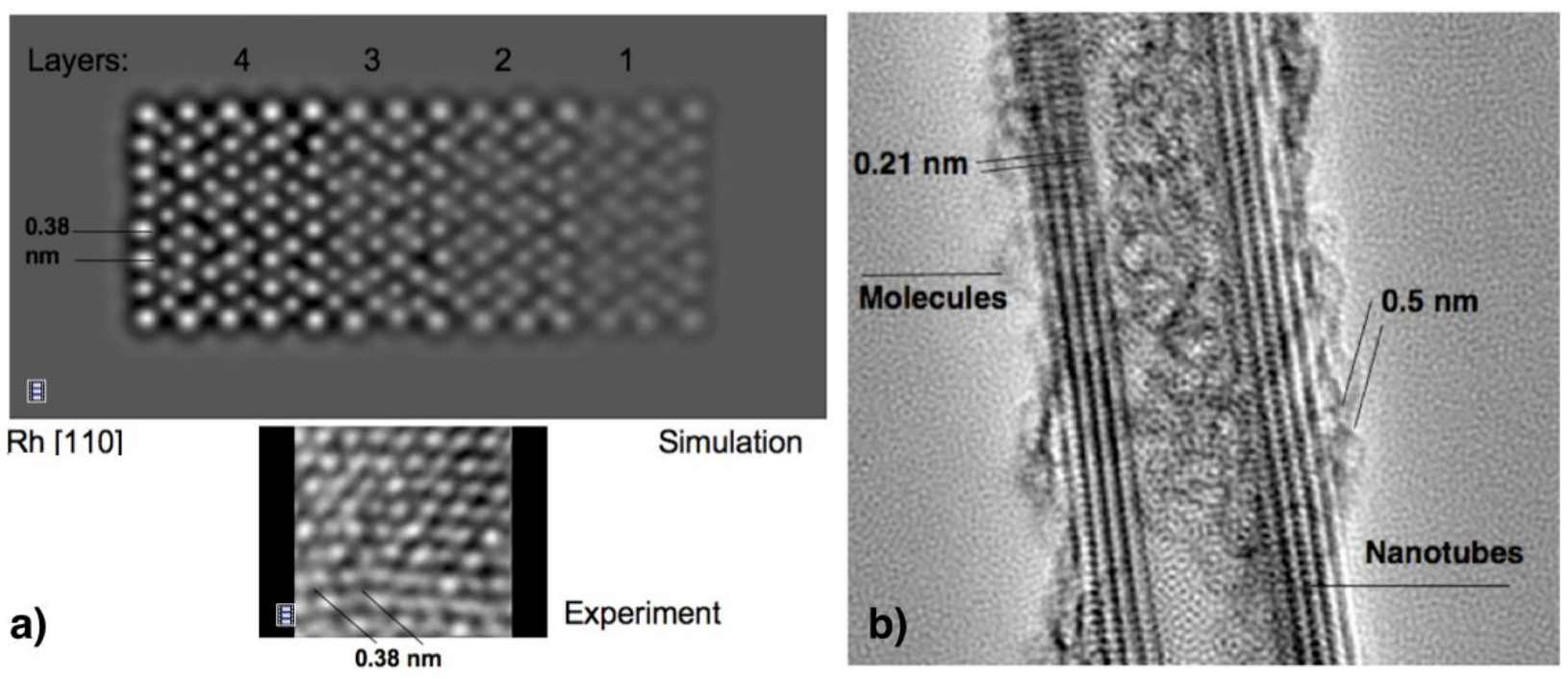
Figure 9:

Single atom dynamics

a) Fourier transform of a clean single layer graphene . b) Fourier transform of a single layer graphene with amorphous hydrocarbon contaminations attached. Fourier components from the supporting graphene layer are removed by a mask. c) Inverse Fourier transform of 3 successive images where Fourier components from supporting graphene film were removed as in b). The magnified images allow studying the time evolution of bond arrangements in amorphous structures with single atom sensitivity. It is observed how local patches of graphene emerge (dotted box) from random atom distributions.

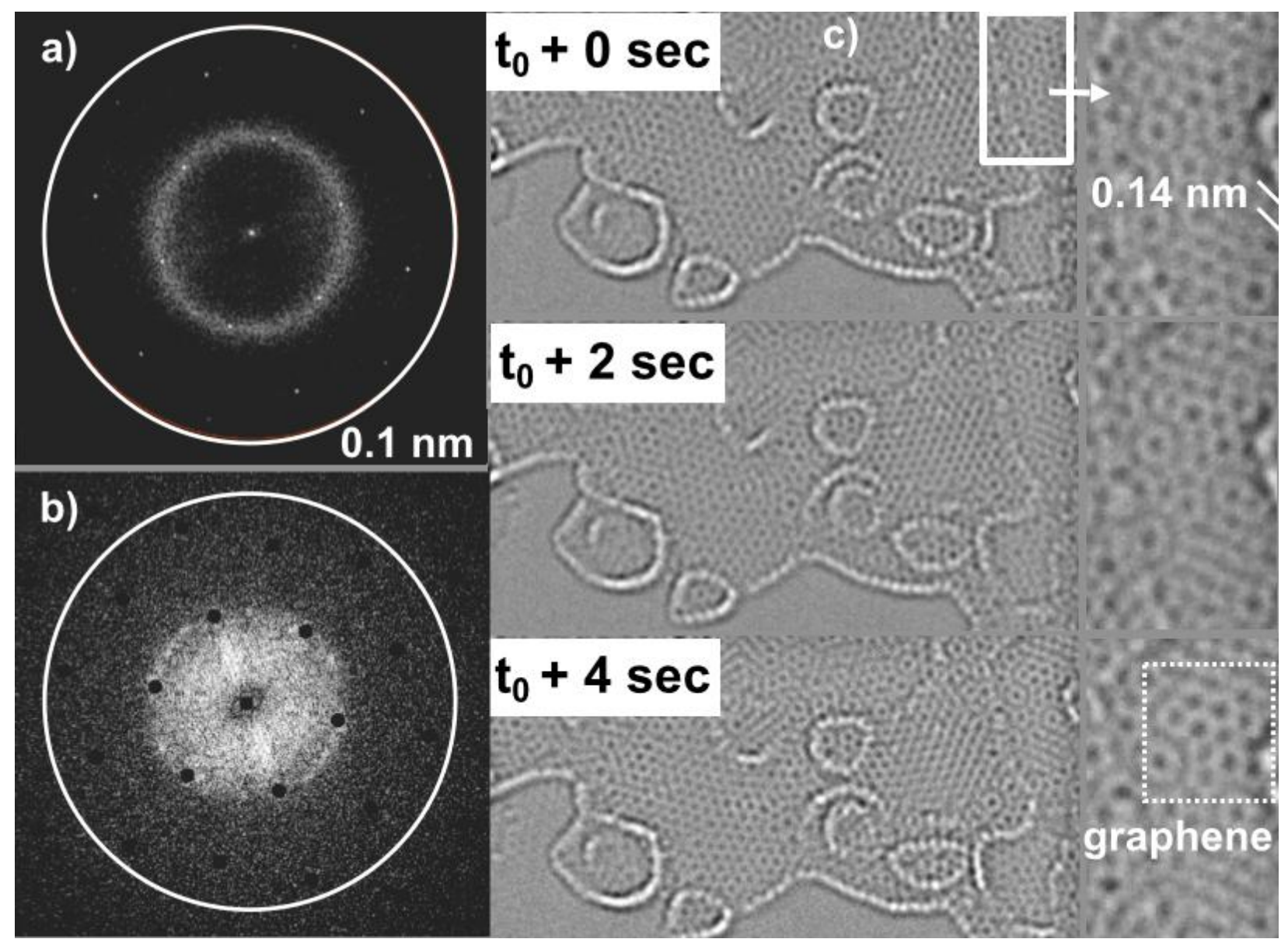

